\title{
Modeling of Correlated Two-Dimensional Non-Gaussian Noises
}

\author{
Vladimir Mikhailovich Artuschenko ${ }^{1}, \mathrm{Kim}$ Leonidovich Samarov ${ }^{2}$, Andrey Petrovich Golubev ${ }^{1}$, Aleksey \\ Yurievich Shchikanov ${ }^{1} \&$ Aleksey Sergeevich Kochetkov ${ }^{1}$ \\ ${ }^{1}$ The Federal State-Funded Educational Institution of Higher Professional Education Russian State University of \\ Tourism and Service, Russia \\ 2 The State-Funded Educational Institution of Higher Professional Education Moscow region Finance and \\ Technology Academy, 42, Gagarina street, Korolev city, Moscow region, Russia \\ Correspondence: Aleksey Sergeevich Kochetkov, The Federal State-Funded Educational Institution of Higher \\ Professional Education Russian State University of Tourism And Service, 99, Glavnaya Street, Cherkizovo \\ village, Pushkinskiy district, Moscow region, 141221, Russia.
}

Received: September 10, 2014

Accepted: September 25, 2014

Online Published: July 27, 2015

doi:10.5539/mas.v9n8p300

URL: http://dx.doi.org/10.5539/mas.v9n8p300

\begin{abstract}
The article describes and analyzes mathematical models of multiplicative and additive non-Gaussian noises affecting the useful signals. For synthesis and analysis, and, hence, the effective design of information systems and radio devices operating in conditions of intense perturbations it is necessary to choose not only the adequate mathematical model of the useful signals and information processes, but also the corresponding models of random effects, possessing in general non-Gaussian multiplicative and additive character. To describe the arbitrary non-Gaussian noises, which are quasiharmonic processes whose spectrum is close (or narrowband) to the band of the desired signal, the authors used a two-dimensional elliptic symmetric probability density function, including two extreme cases: Gaussian processes and a sinusoidal signal with random initial phase distributed uniformly in the interval $[0,2 \pi]$. Model of correlated non-Gaussian narrowband noises of elliptically symmetric two-dimensional probability density function allows you to make a synthesis of information systems and devices based only on a priori knowledge of one-dimensional probability density function and the correlation function. Because knowing one-dimensional probability density function of the instantaneous values, we can determine the probability density function of the envelope; this makes it possible to use the elliptically symmetric probability density function to describe not only additive, but multiplicative (baseband) noises. To describe the real density of probability density function of the non-Gaussian process, the authors propose to approximate its a priori known one-dimensional probability density function and a specially designed transitional probability density function, and show the adequacy of this approximation of the real two-dimensional probability density function of correlated noises.
\end{abstract}

Keywords: information process, adequate mathematical models, additive and multiplicative noise, the probability density function, non-Gaussian processes

\section{Introduction}

For synthesis and analysis, and, consequently, for efficient design of radio systems and devices operating under intense perturbations, it is necessary to select not only adequate mathematical models of information processes $\lambda(t)$, but also accidental impacts, having, in general, the multiplicative $\eta(t)$ and additive $n(t)$ character (Artyushenko and Volovach, 2013; Artyushenko, 2013; Artyushenko and Samarov, 2013). Typically, the disturbance (noise) acting on radio systems and devices, are random processes with non-Gaussian probability density function (PDF) (stationary and non-stationary) (Artyushenko and Abbasova, 2011; Yong and Westerberg, 1971; Edward and Wegman, 1983; Bucy and Mallinckrodt, 1973). The most complete description of stochastic processes (sequences) is a method of multidimensional PDF. Several methods for describing and modeling of stochastic processes with multidimensional PDF. One of such methods is the mixing of random processes (Trofimov, 1986), based on the concept of a PDF random sequence $\left\{\lambda_{h}, h=\overline{1 . H}\right\}$ by the sum of weighted PDF: $W\left(\lambda_{1}, \ldots, \lambda_{H}\right)=\sum_{i=1}^{N} p_{i} W_{i}\left(\lambda_{1}, \ldots, \lambda_{H}\right)$, where $p_{i}$ is random weighting coefficients, provided that $\sum_{i=1}^{N} p_{i}=1 ; W_{i}\left(\lambda_{1}, \ldots, \lambda_{H}\right)$ - given H-dimensional distributions.

Sequence elements $\left\{\lambda_{h}, h=\overline{1 . H}\right\}$ are interpreted as samples obtained by discretization of the corresponding 
process $\lambda(t)$ at the time moment $t_{h}$, provided that, generally, $t_{h}-t_{h-1}=T_{\mathrm{o}}=$ const. The most widely used case is when as $W_{i}\left(\lambda_{1}, \ldots, \lambda_{H}\right)$ there are used $\mathrm{N}$-dimensional normal distributions.

Great opportunities for receiving multidimensional PDFs open Markov processes, allowing with the required accuracy to approximate the random process. In this article we consider the continuous-valued Markov processes.

\section{Methodology}

As is known, a common form of description of random Markov processes is the systems of statistical differential equations, as well as shaping filters. The complexity of the formation and the need to set a large number of a priori information, which is often difficult to obtain in practice (especially for non-Gaussian PDF), sometimes forced to abandon a full probabilistic description of random processes in favor of simplified. Most available information on any random process is one-dimensional correlation function and PDF. In these circumstances, to describe the real information and noises, there are widely used Markov models. Their high efficiency is widely known from the works about the Markov theory of nonlinear filtering (Yarlykov, 1980; Tikhonov and Mironov, 1977).

To describe arbitrary non-Gaussian noises, which are quasiharmonic processes whose spectrum is close (or narrowband) with a band of the desired signal, there can be used elliptical symmetric (ES) two-dimensional PDFs, including two extreme cases: Gaussian processes and a sinusoidal signal with random initial phase

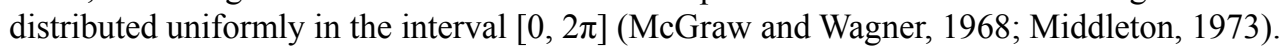

Elliptically symmetric two-dimensional PDFs $W_{2}\left(n_{1}, n_{2}\right)$ of the stationary process $n(t)$ depend on $n_{1}$ and $n_{2}\left(n_{1}=\right.$ $\left.n(t), n_{2}=n(t+\tau)\right)$ only in the combination $l=\left[n_{1}^{2}+n_{2}^{2}-2 r(\tau) n_{1} n_{2}\right]^{0.5}$, where $r(\tau)=B_{n}(\tau) / B_{n}(0)$ is a correlation coefficient of values $n_{1}$ and $n_{2}$, represents a form of ellipses.

Consequently, it is possible to write that

where $C$ is a normalization constant; $R=l\left(1-r^{2}\right)^{-0.5}$;

$$
W_{2}\left(n_{1}, n_{2}\right)=C f(R),
$$

$$
f(R)=\left[2 \pi\left(1-r^{2}\right)\right]^{-1} \int_{0}^{\infty} \Theta(v) J_{0}(R v) v d v
$$

- function is the transformation zero-order Bessel-dimensional characteristic function $\Theta(v)$ of the random process being under the consideration.

As it can be seen from the relations (1), (2), $W_{2}\left(n_{1}, n_{2}\right)$ is completely determined by the one-dimensional PDF $W_{1}(n)$, related to the Fourier transformation with the characteristic function $\Theta(v)$ and a correlation coefficient $r(\tau)$ of the process being under the consideration. At the same time the one-dimensional PDF and the corresponding characteristic function are the even functions.

However, it should be noted that for the construction of ES-distribution (1), there may be used only such even functions $W_{1}(n)$, that lead to a non-negative and integrable function $W_{2}\left(n_{1}, n_{2}\right)$.

In this case fulfillment of the inequality $\int_{0}^{R} R f(R) d R<\infty$, is a necessary and sufficient condition for the existence of ES two-dimensional distribution determined through $W_{1}(n)$ and $r(\tau)$ (Middleton, 1973).

Function

$$
W(R)=2 \pi \mathrm{C}\left(1-r^{2}\right)^{0.5} R f(R),
$$

when describing the narrowband random process coincides with the PDF envelope (amplitude U) of the process. Therefore, the expression (3) can be written as:

$$
W(U)=2 \pi \mathrm{C}\left(1-r^{2}\right)^{0.5} R f(U) .
$$

It is a feature of ES distributions arising from their definition (Trofimov, 1986). As for the noises with a band spectrum the probability density function of amplitude (PDFA) is a sufficiently probable characteristic, so that it can be argued that the ES model of the corrected non-Gaussian process uniquely determines such noises.

In (Middleton, 1973; Artyushenko and Solenov, 1993) there are presented the main characteristics of random processes, which two-dimensional distributions possess elliptical symmetry.

Note that when adding arbitrary ES processes to the same correlation coefficients, the obtained process is also the ES process.

So, when you add a sinusoid to random initial phase uniformly distributed in the interval $[0,2 \pi]$, and narrowband Gaussian noise (at the equal correlation coefficients) taking into account the relations (1), (2) we get 
the ES process, which PDFA is subject to the Rice law:

$$
W_{2}\left(n_{1}, n_{2}\right)=\left[2 \pi\left(1-r^{2}\right)^{0.5} \sigma^{2}\right]^{-1} \exp \left\{\left(U^{2}+R^{2}\right)\left(2 \sigma^{-1}\right)\right\} I_{0}\left(U R \sigma^{-2}\right)
$$

where $U$ is the amplitude of the sinusoidal component; $\sigma^{2}$ is the noise variance; $r(\tau)=\cos \omega_{0} \tau$.

You can come to the PDF described by (4) by using the relation (2) and the expression of the characteristic function for the total process:

$$
\Theta_{1}(v)=J_{0}(U v) \exp \left\{-0,5 \sigma^{2} v^{2}\right\}
$$

By integrating (2) we obtain (4).

Note that in the case of an arbitrary correlation $r(\tau)=r_{0}(\tau) \cos \omega_{0} \tau$, where $r_{0}(\tau)$ is a slowly decaying function, described by the expression (5), there can be no ES. In this case, the two-dimensional PDF (4) can be considered as a ES model provided that $\tau<<\tau_{\text {cor }}$, where $\tau_{\text {cor }}$ is a correlation interval of the describing processes defined by the envelope $r(\tau)$ (Artyushenko and Solenov, 1993).

Let us consider as an example the case when the instantaneous values of additive noise described by generalized Gaussian distribution:

$$
\begin{gathered}
W(n)=\left[v \gamma\left(\sigma_{n}, v\right) / 2 \Gamma\left(v^{-1}\right)\right] \exp \left\{-\left[\gamma\left(\sigma_{n}, v\right)|n|\right]^{v}\right\}, \\
\text { where } \gamma\left(\sigma_{n}, v\right)=\sigma_{n}^{-1}[\Gamma(3 / v) / \Gamma(1 / v)]^{0.5} .
\end{gathered}
$$

Considering that matching sampling time moments of quadrature components are uncorrelated, we obtain:

$$
\begin{gathered}
W_{2}\left(n_{1}, n_{2}\right)=\left[v \gamma_{0}^{2}\left(\sigma_{n}, v\right) / 2 \pi \Gamma(2 / v)\right] \exp \left\{-\left[\gamma_{0}\left(\sigma_{n}, v\right)\left(n_{1}{ }^{2}+n_{2}^{2}\right)^{0.5}\right]^{v}\right\}, \\
\text { where } \gamma_{0}\left(\sigma_{n}, v\right)=\sigma_{n}{ }^{-1}[\Gamma(4 / v) / 2 \Gamma(2 / v)]^{0.5} ;-\infty<n_{1} ; n_{2}<\infty .
\end{gathered}
$$

At the same time the PDF noises:

$$
W(U)=\left[v \gamma_{0}\left(\sigma_{n}, v\right) U / 2 \Gamma(2 / v)\right] \exp \left\{-\left[\gamma_{0}\left(\sigma_{n}, v\right) U\right]^{v}\right\} ; 0 \leq U<\infty .
$$

If the narrow-band random process is stationary, then PDFA $W(U)$ and PDF of its instantaneous values are interrelated by (Artyushenko and Solenov, 1993):

$$
W(U)=U \int_{0}^{\infty} v \Theta_{n}(v) J_{0}(U) d v,
$$

where $\Theta_{n}(v)=\int_{-\infty}^{\infty} W(n) \exp \{j v n\} d n$ is a characteristic function of the process $n(t)=U(t) \cos \mathrm{F}(t) ; U(t)$ and $\mathrm{F}(t)$ are the envelope and the total phase of a random process, respectively.

Making the necessary changes to (6), we obtain:

$$
W(n)=\pi^{-1} \int_{|n|}^{\infty} W(U)\left(U^{2}-n^{2}\right)^{-0.5} d U .
$$

Thus, the description of correlated non-Gaussian narrowband noise of elliptically symmetric two-dimensional PDF allows to produce synthesis systems and devices based on a priori knowledge and one-dimensional PDF and correlation function. Knowing the one-dimensional PDF of instantaneous values $W(n)$, we can determine the PDF of the envelope $W\left(\mathrm{U}_{n}\right)$, which makes it possible to use an elliptically symmetrical PDF for description of a multiplicative (modeling) noises.

Along with the described above methods for description of correlated non-Gaussian processes, let us consider the following.

The real PDF of non-Gaussian process, it is proposed to approximate by a priori known one-dimensional PDF $W\left(n_{h-1}\right)$ and specially designed transitional PDF $W^{A}\left(n_{h} \mid n_{h-1}\right)$. As a result the PDF of non-Gaussian process will be described as:

$$
W^{A}\left(n_{h}, n_{h-1}\right)=W\left(n_{h}\right) W^{A}\left(n_{h} \mid n_{h-1}\right) .
$$

As a transitional PDF, we use the PDF of the following form:

$$
W^{A}\left(n_{h} \mid n_{h-1}\right)=\left(2 \pi G^{2}\right)^{-0.5} \exp \left\{-\left[n_{h}-\mathrm{M}\left(n_{h-1}\right)\right]^{2} / 2 G^{2}\right\},
$$

where $G^{2}$ characterizes the intensity of the random process $\left\{n_{h}\right\}$;

$\mathrm{M}\left(n_{h-1}\right)=n_{h-1}-0,5 G^{2} \frac{d}{d n_{h-1}} \ln W\left(n_{h-1}\right)$ is a function of a special form.

Note that in case when the process $\left\{n_{h}\right\}$ is described by a Gaussian PDF $W\left(n_{h-1}\right)=N\left(0, \sigma^{2}\right)$, equation (8) takes the form of:

$$
W^{A}\left(n_{h} \mid n_{h-1}\right)=\left(2 \pi G^{2}\right)^{-0.5} \exp \left\{-\left(n_{h}-r n_{h-1}\right)^{2} / 2 G^{2}\right\} .
$$


Considering $G^{2}=\sigma^{2}\left(1-r^{2}\right)$, come to the well-known expression for the conditional Gaussian PDF.

Representation of the two-dimensional PDF in the form (9) will be used further in the synthesis tasks, so it is necessary to substantiate the adequacy of the introduced approximation.

As a criterion we will use the information criterion:

$$
\min I_{K}\left(W, W^{A}\right) ; n_{h}, n_{h-1} \in \Pi,
$$

where $I_{K}$ is the Kullback information characterizing the estimate of the average information, containing in the area $\Pi$ of changes the component $n_{h}$ and $n_{h-1}$, correlated random process at hypotheses testing

$$
\mathrm{H}_{0}: W\left(n_{h} \mid n_{h-1}\right) \text { and } \mathrm{H}_{1}: W^{A}\left(n_{h} \mid n_{h-1}\right) \text {. }
$$

There are two methods for the appraisal of the Kullback:

$$
\begin{aligned}
& I_{12 \mathrm{~K}}\left(W, W^{A}\right)=\iint_{-\infty}^{\infty} W\left(n_{h}, n_{h-1}\right) \ln \frac{W\left(n_{h}, n_{h-1}\right)}{W^{A}\left(n_{h}, n_{h-1}\right)} d n_{h} d n_{h-1} \\
& I_{21 \mathrm{~K}}\left(W, W^{A}\right)=\iint_{-\infty}^{\infty} W^{A}\left(n_{h}, n_{h-1}\right) \ln \frac{W^{A}\left(n_{h}, n_{h-1}\right)}{W\left(n_{h}, n_{h-1}\right)} d n_{h} d n_{h-1} .
\end{aligned}
$$

Criterion (10) and relations (11), (12) will be used at the testing phase when checking validity of PDF non-Gaussian processes PRB description made by the relations (7) and (8).

Let us consider a few examples of non-Gaussian processes for which $W\left(n_{h}, n_{h-1}\right)$ is known, and then, turn to the description of non-Gaussian correlated processes for which are known only the one-dimensional PDFs.

As testing PDFs, we introduce the distribution of the form:

$$
\begin{gathered}
W\left(n_{h}\right)=\frac{v}{\Gamma\left(v^{-1}\right) \sigma}\left[\frac{\Gamma(3 / v)}{\Gamma\left(v^{-1}\right)}\right] \exp \left\{-\left[\frac{\Gamma(3 / v)}{\Gamma\left(v^{-1}\right)}\right]^{v / 2}\left[\frac{\left|n_{h}\right|^{v}}{\sigma^{v}}\right]\right\}, v \geq 0,5 \\
W\left(n_{h} \mid n_{h-1}\right)=\frac{v}{2 \Gamma\left(v^{-1}\right) \sigma}\left[\frac{\Gamma(3 / v)}{\left(1-r^{2}\right) \Gamma\left(v^{-1}\right)}\right]^{0.5} \exp \left\{-\left[\frac{\Gamma(3 / v)}{\left(1-r^{2}\right) \Gamma\left(v^{-1}\right)}\right]^{v / 2}\left[\frac{\left|n_{h}-r n_{h-1}\right|^{v}}{\sigma^{v}}\right]\right\},
\end{gathered}
$$

where $\Gamma($.$) is a gamma function.$

Note that as special cases of (13) and (14), there follows a Gaussian distribution (9) and PDF of Laplace, consequently at $v=2$ and $v=1$.

Following the procedure outlined above, as a transitional approximating $W^{A}\left(n_{h} \mid n_{h-1}\right)$ for PDF of the considered form we will have:

$W^{A}\left(n_{h} \mid n_{h-1}\right)=\left(2 \pi G^{2}\right)^{-0.5} \exp \left\{-\left[n_{h}-\mathrm{M}\left(n_{h-1}\right)\right]^{2} / G^{2}\right\}$,

where $\mathrm{M}\left(n_{h-1}\right)=n_{h-1}-0,5 G^{2} \mathrm{Z}_{\mathrm{A}}\left(n_{h-1}\right)$;

$$
\mathrm{Z}_{\mathrm{A}}\left(n_{h-1}\right)=-\frac{d}{d n_{h-1}} \ln W\left(n_{h-1}\right)=\frac{v}{2^{0.5 v} \sigma^{v}}\left|n_{h-1}\right|^{v-1} \operatorname{sgn}\left(n_{h-1}\right) .
$$

Let us consider the example of the construction of two-dimensional PDFs of non-Gaussian process in accordance with the methodology set forth, if there is known only the true one-dimensional PDF $W\left(n_{h-1}\right)$ of the form (13).

In accordance with (8) let us define the transitional PDF. Following (15), we write the function $\mathrm{M}\left(n_{h-1}\right)$ :

$$
\mathrm{M}\left(n_{h-1}\right)=n_{h-1}-0,5 G^{2} \frac{d}{d n_{h-1}}\left\{-\left[\frac{\Gamma(3 / v)}{\Gamma\left(v^{-1}\right)}\right]^{v / 2}\left[\frac{\left|n_{h-1}\right|^{\nu}}{\sigma^{v}}\right]\right\}=n_{h-1}-0,5 G^{2} \frac{v}{2^{0.5 v} \sigma^{v}}\left|n_{h-1}\right|^{v-1} \operatorname{sgn}\left(n_{h-1}\right) .
$$

By introducing for the computational convenience the concept of equivalent correlation coefficient $r_{3}$, defined from the relation $G^{2} / \sigma^{2}=1-r_{3}$, we finally write the expression of the transitional PDF:

$$
\begin{gathered}
W^{A}\left(n_{h} \mid n_{h-1}\right)=\left[2 \pi \sigma^{2}\left(1-r_{\ni}\right)\right]^{-0.5} \exp \left\{\frac{\left[n_{h^{-}} n_{h-1}+0.5 \sigma^{2}\left(1-r_{\ni}\right) z_{\ni}\left(n_{h-1}\right)\right]^{2}}{\sigma^{2}\left(1-r_{\ni}\right)}\right\}, \\
\text { where } z_{\ni}\left(n_{h-1}\right)=\frac{v}{2^{0.5 v} \sigma^{\nu}}\left|n_{h-1}\right|^{v-1} \operatorname{sgn}\left(n_{h-1}\right) .
\end{gathered}
$$

Results. The results of modeling the two-dimensional non-Gaussian PDFs in accordance with relations (7), (16) and (13) for different correlation coefficients $r_{\ni}$ and $v$ are shown in the Figure 1. 

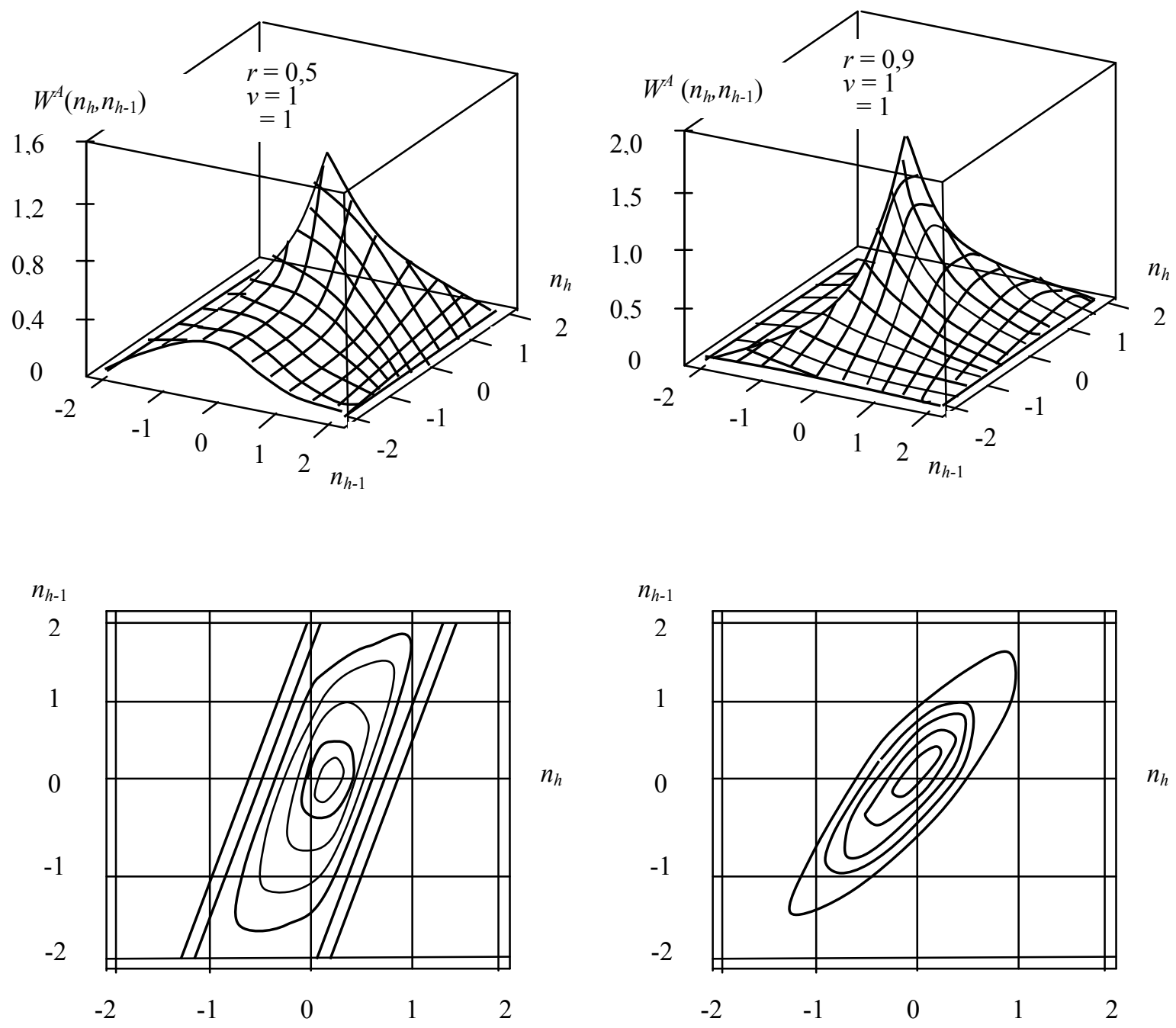

a)

b)
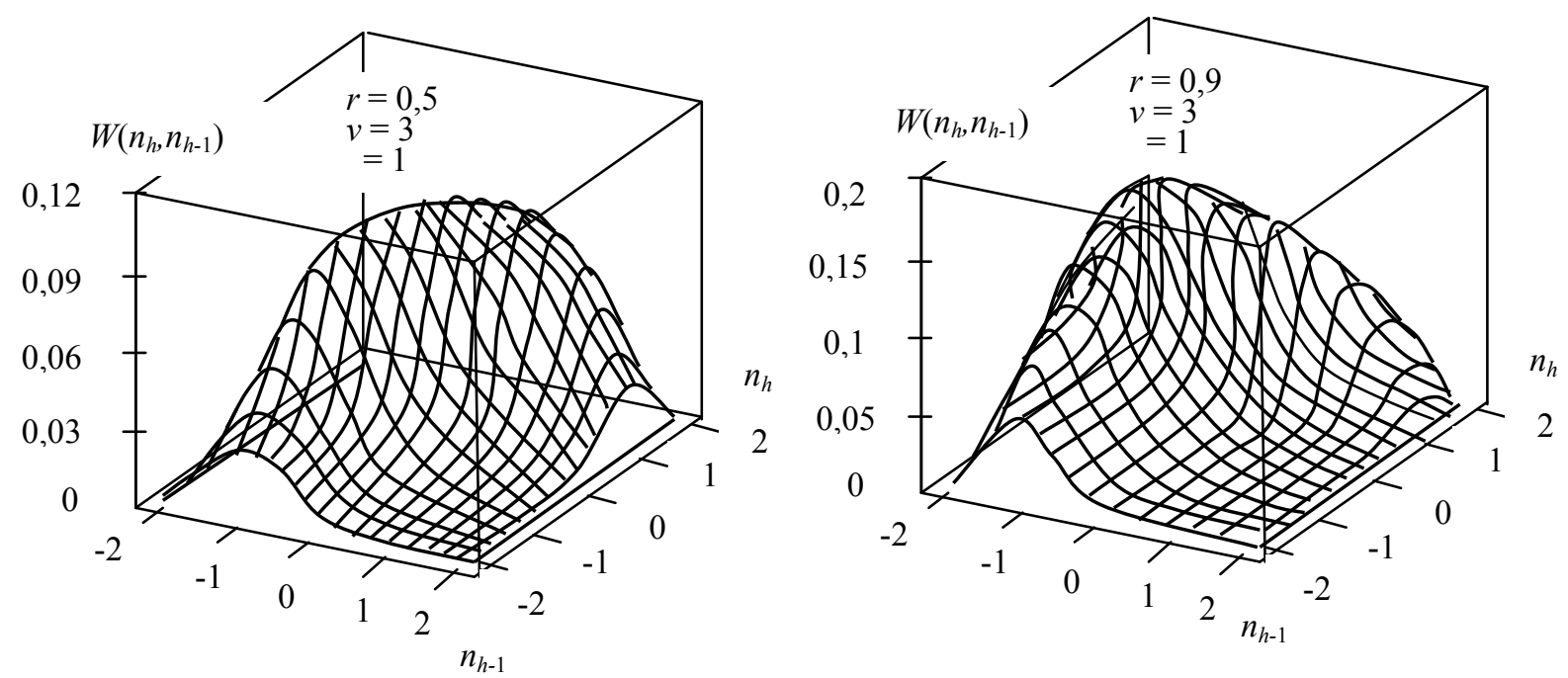


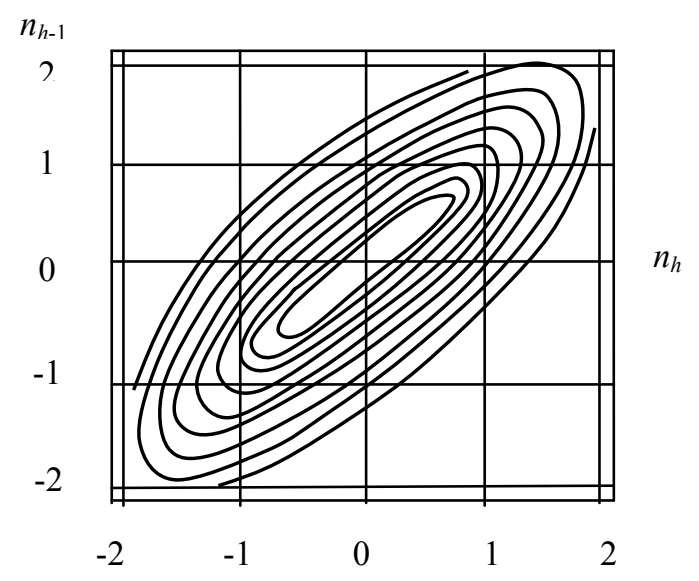

c)

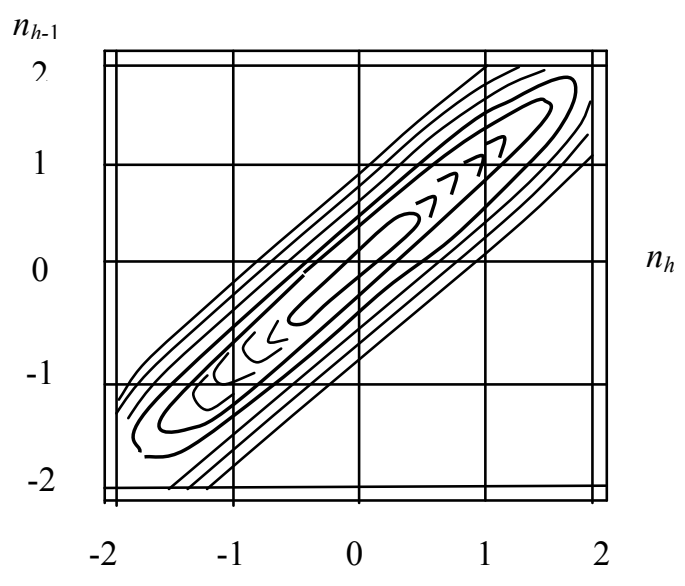

d)

Figure 1. The results of modeling the two-dimensional non-Gaussian PDFs and isolines of their surfaces at: $\mathrm{a}-\mathrm{r}$ $=0,5, v=1 ; \mathrm{b}-\mathrm{r}=0,9, v=1 ; \mathrm{c}-\mathrm{r}=0,5, v=3 ; \mathrm{d}-\mathrm{r}=0,9, v=3$

The analysis presented in PDF Fig. 1 shows that at large correlation coefficients the approximating $W^{A}\left(n_{h}, n_{h-1}\right)$ and true $W\left(n_{h}, n_{h-1}\right)$ PDFs are approaching each other. However, to accurately determine the degree of similarity of these distributions we use quantitative assessment of PDF similarity measure (10), (11), (1.28). Restrict ourselves to a particular case of PDF (14) - PDF of Laplace, which takes place at $v=1$.

Graphs of dependancy $I_{12 \mathrm{~K}}$ and $I_{21 \mathrm{~K}}$ are shown in the Fig. $2 a$ and Fig. $2 a$, respectively. Fig. $2 a$ and Fig. $2 b$ show the lines of equal level of displayed surfaces. The most informative surface, as seen from Fig. 2 and 3, is the surface $I_{21 \mathrm{~K}}\left(n_{h}, n_{h-1}\right)$, which illustrates that with increasing $r$ and $r_{\ni}$ grows the degree of PDF closeness.

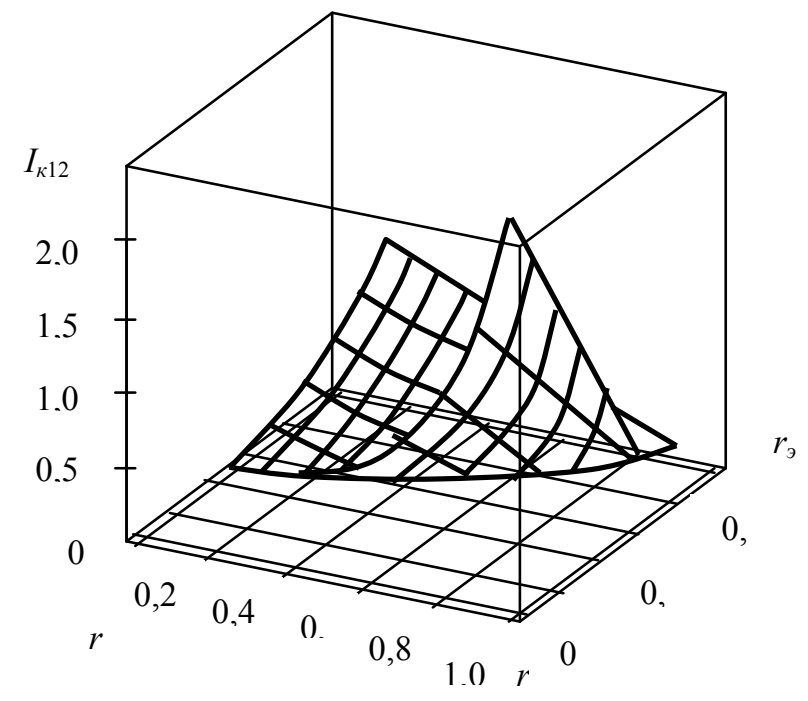

a) 


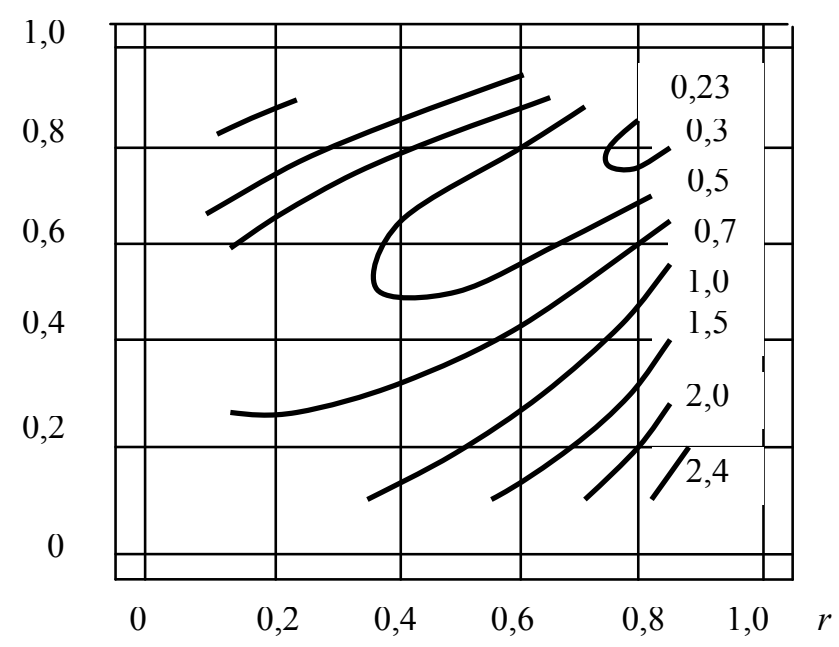

b)

Figure 2. Dependences Ik12 $=\mathrm{f}(\mathrm{r}, \mathrm{r} \ni)$ and isolines of their surfaces
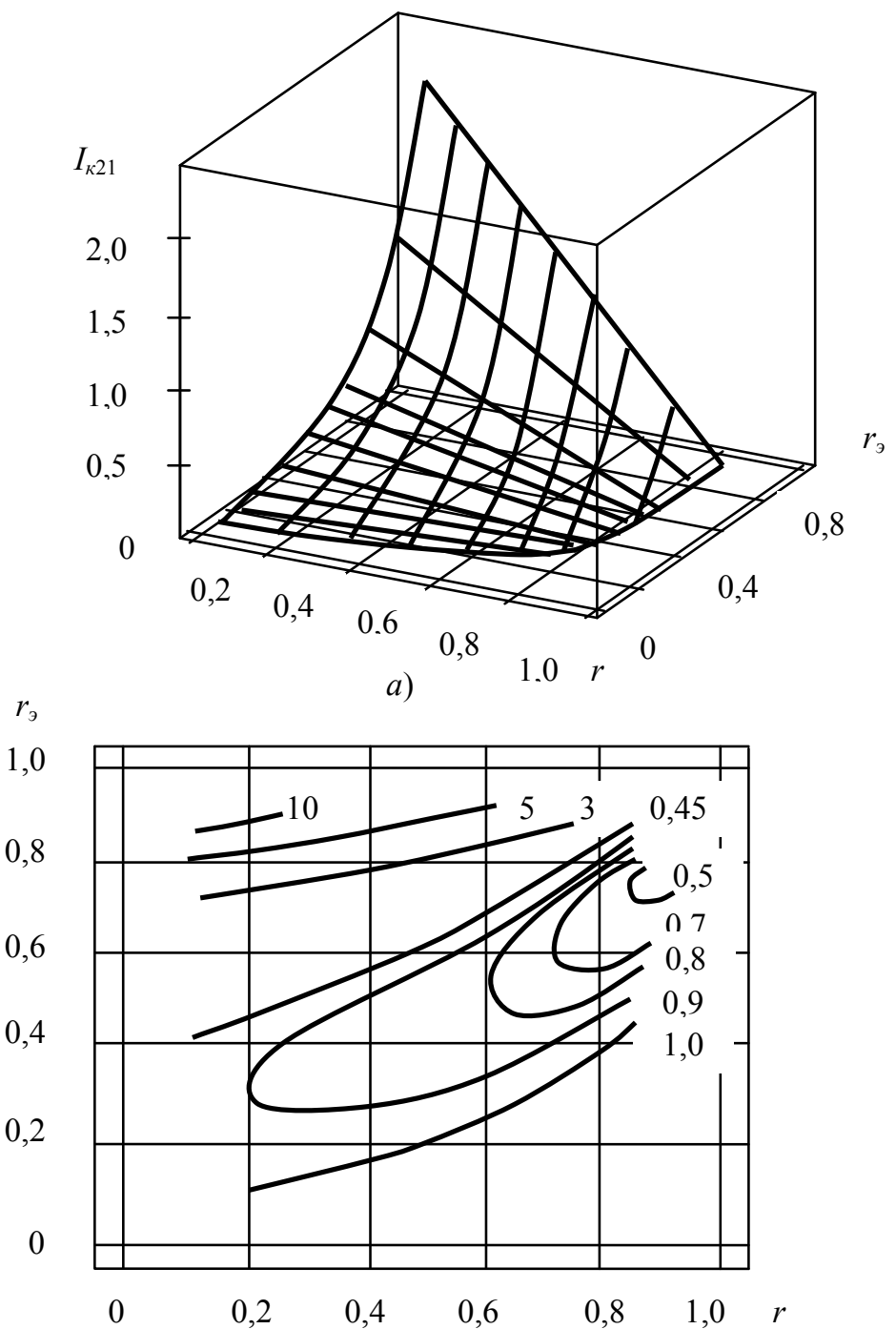

b)

Figure 3. Dependencies Ik21 $=f(r, r \ni)$ and isolines of their surfaces 


\section{Discussion}

Along with information on the Kullback, the wide practical importance, especially in problems of statistical synthesis of optimal algorithms for processing, received the information by Fisher, contained in a random process with PDFs $W(n)$ (Trofimov, 1986):

$$
I_{\mathrm{F}}=\left\{m\left[\frac{d \ln W(n)}{d n}\right]^{2}\right\}=\int_{-\infty}^{\infty}\left[\frac{d \ln W(n)}{d n}\right] W(n) d n .
$$

It can be shown that in the case of one-dimensional PDFs, we have the identity: $m\left\{\frac{d^{2} \ln W(n)}{d n^{2}}\right\}=-m\left\{\left[\frac{d \ln W(n)}{d n}\right]^{2}\right\}$.

In case the random process is defined by the two-dimensional $W\left(n_{1}, n_{2}\right)$ or conditional $W\left(n_{1} \mid n_{2}\right)$ PDF by analogy with $I_{\mathrm{F}}$, there is introduced the concept of the Fisher information matrix $I_{\mathrm{F}}$ with the elements:

$$
I_{\mathrm{F} . j j}=m\left\{\frac{d \ln W\left(n_{1} \mid n_{2}\right)}{d n_{i}} \frac{d \ln W\left(n_{1} \mid n_{2}\right)}{d n_{j}}\right\}=\iint_{-\infty}^{\infty} \frac{d^{2} \ln W\left(n_{1} \mid n_{2}\right)}{d n_{i} d n_{j}} \ln W\left(n_{1}, n_{2}\right) d n_{1} d n_{2} ; i, j=1,2 .
$$

It is supposed that the matrix $\left\|I_{\Phi}\right\|$ positively defined, i. e. $\operatorname{det} I_{\mathrm{F}} \neq 0$.

In the particular case of a Gaussian random process $n(t)$, defined transition distribution, the Fisher information matrix obtains the form of:

$$
\left\|I_{\Phi . \Gamma}\right\|=\left\|\begin{array}{ll}
I_{\Phi .11} & I_{\Phi .12} \\
I_{\Phi .21} & I_{\Phi .22}
\end{array}\right\|=\left[\sigma_{n}^{2}\left(1-r_{n}^{2}\right)\right]^{-1}\left\|\begin{array}{cc}
1 & -r_{n} \\
-r_{n} & r_{n}^{2}
\end{array}\right\|,
$$

where $\sigma_{n}^{2}$ is a variance, and $r_{n}$ is a correlation coefficient of the random process $n(t)$.

From a comparison of the member $I_{\mathrm{F} .11}$ and $I_{\mathrm{F}}$ for PDF with independent values it follows that the factor at the | right part $I_{\mathrm{F} .11}$ at $r_{n}=0$ coincides with $I_{\mathrm{F}}$ for the Gaussian PDF. While $0 \leq r_{n}^{2}<1$, it becomes clear that existence of a finite correlation between the values $n(t)$ results in an increase of Fischer information compared with the case of one-dimensional PDF (Artyushenko and Samarov, 2013).

In general, the definition of the information matrix for non-Gaussian correlation processes encounters considerable difficulties and, as a rule, cannot be obtained analytically. Only in some cases, the solution is possible to obtain analytically. For example, for the PDFs (14) information matrix takes the form:

$$
\left\|I_{\Phi}\right\|=\frac{v(v-1) \Gamma(3 / v) \Gamma\left(1-v^{-1}\right)}{\sigma_{n}^{2} \Gamma^{2}(1 / v)\left(1-r_{n}^{2}\right)}\left\|\begin{array}{cc}
1 & -r_{n} \\
-r_{n} & r_{n}^{2}
\end{array}\right\|=A(v) I_{\mathrm{F} . \Gamma},
$$

where $A(v)=\frac{v(v-1) \Gamma(3 / v) \Gamma\left(1-v^{-1}\right)}{\Gamma^{2}(1 / v)} ; v \geq 2-$ constant depending on the parameter of distribution.

Dependencies of matrix elements $I_{\mathrm{F} . i j}$ on the PDF parameters are shown in Figure 4.

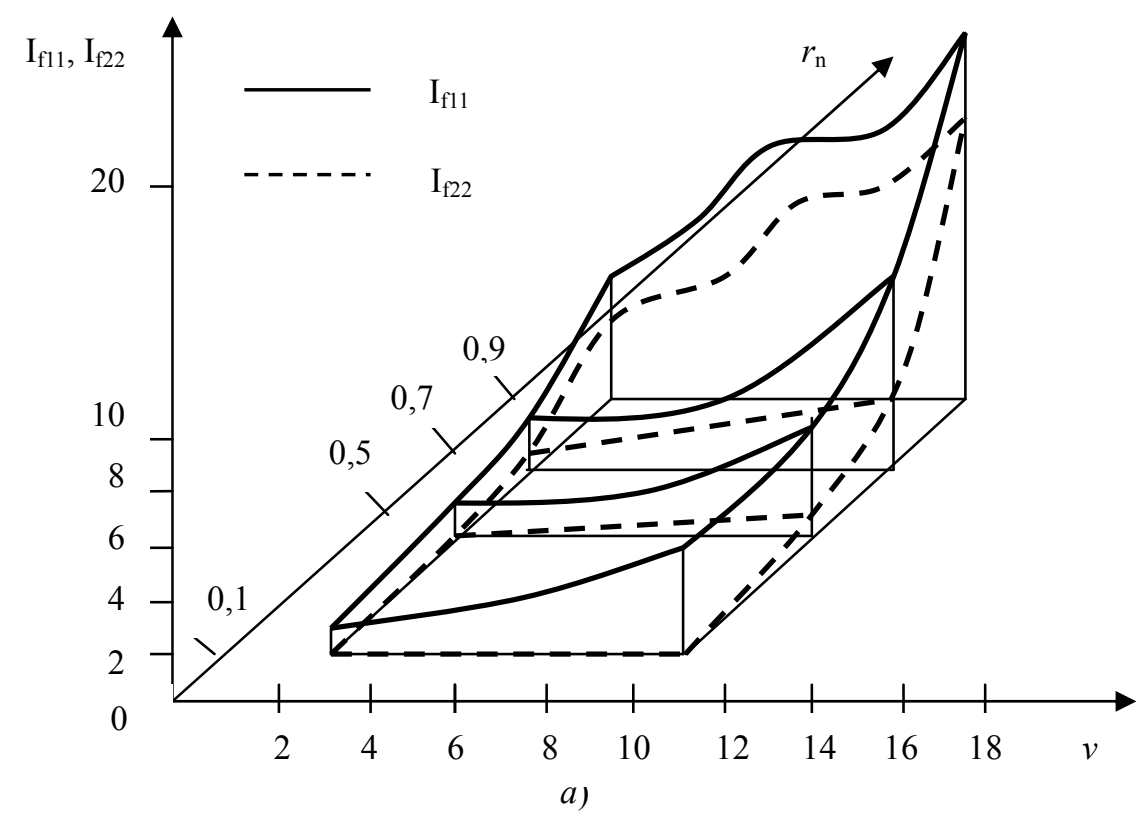




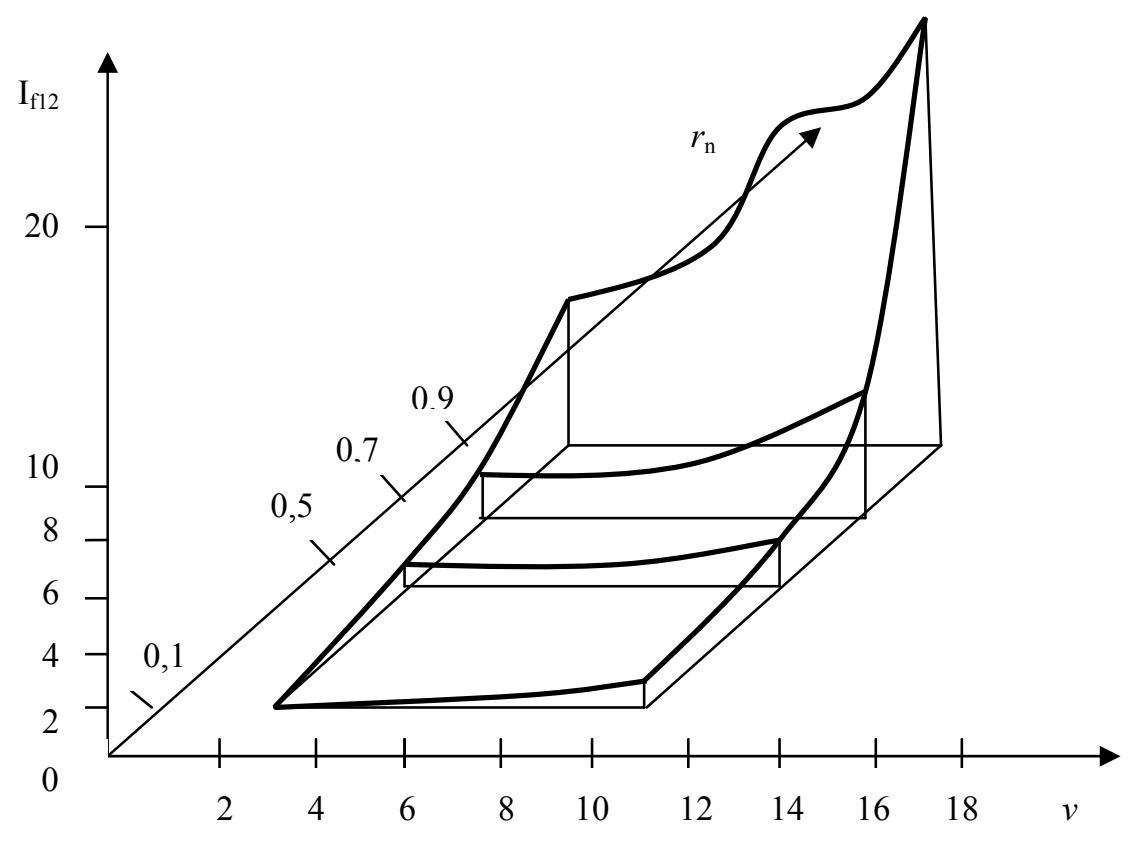

b)

Figure 4. Dependence of matrix elements $\mathrm{I}_{\mathrm{f} i j}$ on the parameters of distribution: $a-\mathrm{I}_{\mathrm{f} 11} \mathrm{I}_{\mathrm{f} 22}$;

$$
b-\mathrm{I}_{\mathrm{f} 12}
$$

Similary to the quasi-Shenon information there is introduced the concept of quasi-Fisher information:

$$
\int_{-\infty}^{\infty} W_{0}(n)\left(\frac{W^{\prime}(n)}{W(n)}\right)^{2} d n=\int_{-\infty}^{\infty} W_{0}(n)\left(\frac{d \ln W(n)}{d n}\right)^{2} d n=m\left\{\left(\frac{W^{\prime}(n)}{W(n)}\right)^{2}\right\}=I_{\Phi}\left(W, W_{0}\right) .
$$

The value $I_{\Phi}\left(W, W_{0}\right)$ is used in particular for the evaluating the effectiveness of the algorithms of asymptotically optimal reception.

Along with the introduced notions of Fisher information in assessing the characteristics of unilateral characteristic PDFs, for example, to describe the random amplitudes of narrowband radio signals, there is used Fisher dispersion information (dinformation) (Tsushkin, 1984; Artyushenko, Volivach and Samarov, 2014; Artyushenko and Volivach, 2013):

$$
I_{\mathrm{F} . \mathrm{D}}(W)=m\left\{n^{2}\left(\frac{W^{\prime}(n)}{W(n)}\right)^{2}\right\}=\int_{-\infty}^{\infty} n^{2}\left(\frac{d \ln W(n)}{d n}\right)^{2} d n .
$$

Note that for the same PDFs the following inequality is performed: $I_{\mathrm{F} . \mathrm{D}}(W)>I_{\mathrm{F}}(W)$.

The theoretical results presented in yielded work have been used by working out and creation of a multipurpose measuring instrument (MMI) of parameters of movement of extended objects, that have past successful tests in actual practice [2]. In its structure the block constructing a multiplicate hindrance has been used, allowed considerably to raise accuracy of work of a measuring instrument. For definition of efficiency again created MMI and existing measuring instruments the experimental researches providing check of their working capacity in a dynamic mode at measurement of speed of movement of extended objects with acceleration to $3 \mathrm{~m} / \mathrm{s} 2$ in a mode of deep stand by regime of Doppler amplitude of a signal (to $80 \%$ from duration of realisations) have been spent. As the examinee of the sample of a radar-tracking measuring instrument speeds (RTMI) was taken a serially produced measuring instrument RTMI-B3. On Figure 5 in uniform systems of co-ordinates schedules norm of errors of measuring instruments are presented $\delta_{\mathrm{o}}=f\left(t_{3 \text { зам }} T_{\mathrm{M}}{ }^{-1} ; F_{д}\right)$, where $t_{\text {зам }} T_{\mathrm{M}}{ }^{-1}$ - relative time of a stand by regime of a processed signal (PS); $T_{\mathrm{M}}=F_{\mathrm{M}}{ }^{-1}, F_{\mathrm{M}}$ - frequency of the signal modulated on amplitude at $100 \%$-s' peak modulations, $F_{\text {д }}$ - frequency (PS) (Doppler frequency), for cases $F_{\mathrm{M}}=0,1 F_{\text {д. }}$. 


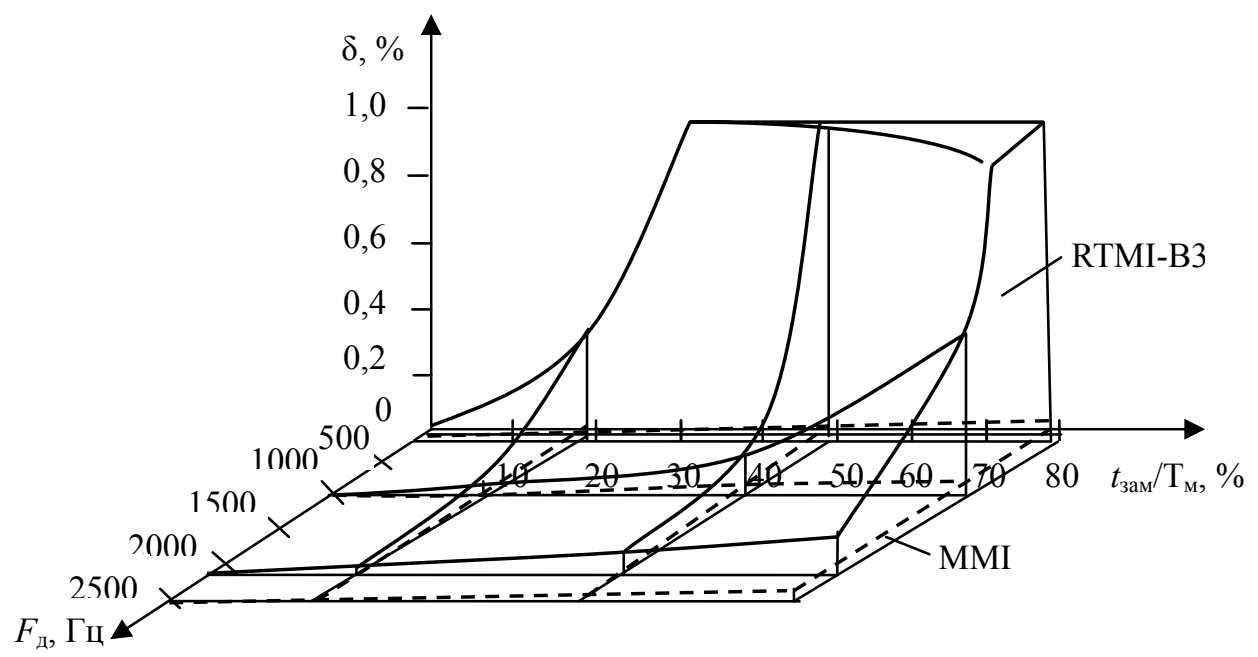

Figure 5. Comparative characteristics norm of errors of measuring instruments

MMI and RTMI-B3

Errors are led to error size of RTMI-B3, where $\delta_{\mathrm{o}}=\delta / \delta_{\mathrm{RTMI-B} 3}$, where $\delta_{\text {RTMI-B3 }}=100 \% ; \delta=\left[\left|F_{\text {д. }}-F_{\text {д.и }}\right| /\right.$ $\left.F_{\text {д.ф }}\right] 100 \%, F_{\text {д.фи }} F_{\text {д.и }}-$ the actual and measured frequency PS.

Apparently from the presented dependences, under identical conditions of measurement of PS, size of relative errors is ruther less, than at RTMI-B3. Fuller results of comparative researches of the created measuring instrument are stated in [2]. Calculation of an estimation of economic efficiency is resulted in (17).

\section{Conclusion}

Thus, there are considered and analyzed mathematical models of multiplicative and additive non-Gaussian noise affecting the useful signals. To perform the synthesis of radio systems and devices, there are introduced elliptical symmetric PDFs, allowing to describe not only narrowband correlated additive noise, but interference with multiplicative (modulating) character.

There is proposed the transitional PDF allowing to design two-dimensional PDFs of correlated non-Gaussian noises. There is shown the adequacy of designed with its help PDFs of two-dimensional real PDFs affecting correlated noise.

There are introduced the information characteristics of non-Gaussian additive and multiplicative noises.

It is shown that in the general case, the definition of information matrix for non-Gaussian correlation processes encounters considerable difficulties and, as a rule, cannot be obtained analytically.

In the future, the considerable interest arises to investigate the possibility of using the Fisher dispersion information to describe the statistical characteristics of multiplicative non-Gaussian noise, significantly affecting the quality of the information processed upon receipt.

Experimental researches created with use of the theoretical results presented in article, a multipurpose measuring instrument of parametres of movement of extended objects, show that an error of measurement at it much more lower than at similar serially produced measuring instruments.

\section{References}

Artushenko, V. M., \& Volovach, V. I. (2012). Estimation of economic efficiency of work of a radar-tracking measuring instrument of speed. The bulletin of Volga region state university of service. A series Economy, 6(26), 182-192.

Artyushenko, V. M. (2013). Research and development of radar meter for motion parameters of extended objects. M., pp: 214.

Artyushenko, V. M., \& Abbasova, T. S. (2011). Designing of multiservice systems under the influence of external electromagnetic noises. M., 264. 
Artyushenko, V. M., \& Samarov, K. L. (2013). Construction of two-dimensional correlated additive and multiplicative models of non-Gaussian noise. Electrical and information complexes and systems, 4(10), 83-92.

Artyushenko, V. M., \& Solenov, V. I. (1993). Elliptically symmetric models of non-Gaussian noise. Kiev, pp. 4-8.

Artyushenko, V. M., \& Volivach, V. I. (2013). Choice and grounding of the models of measured parametres of extended objects motion. School of the university science: Development paradigm, 3(10), 109-113.

Artyushenko, V. M., \& Volovach, V. I. (2013). Statistical Characteristics of Envelope Outliers Duration of non-Gaussian Information Processes. Proceedings of IEEE East-West Design \& Test Symposium (EWDTS'2013). Rostov-on-Don, Russia, September 27-30: 137-140.

Artyushenko, V. M., Volivach, V. I., \& Samarov, K. L. (2014). Construction of two-dimensional correlated models of non-Gaussian noise. Scientific and Technical Herald of the Volga region, 1, 53-56.

Bucy, R. S., \& Mallinckrodt, A. J. (1973). An Optimal Phase Demodulato. Stochastics, 1.

Edward, J., \& Wegman, G. (1983). Tohic in Non-Gaussian Signal Processings. New-York: 235.

McGraw, D. K., \& Wagner, J. F. (1968). Elliptially Symmetric Distributions. IEEE Trans, 1(IT-14), 76-84.

Middleton, D. (1973). Man-madenjise in urban environments and measuzements. Trans. ITTT, 9(AES-9), $371-376$.

Tikhonov, V. I., \& Mironov, M. A. (1977). Markov processes. MA., p. 488.

Trofimov, A. T. (1986). Estimation of nuisance parameters for the adaptive signal processing based on the usage of multi-Gaussian noise model. Radio Engineering and Electronics, 11(31), 2151-2159.

Tsushkin, Y. Z. (1984). Fundamentals of information theory of identification. M., p. 320.

Tzay, Y. Y., \& Westerberg, R. A. (1971). Error bounds for stochastic estimation of signal parameters. IEEE Trans., IT-17, 549-557. http://dx.doi.org/10.1109/TIT.1971.1054696

Yarlykov, M. S. (1980). Application of Markov theory of nonlinear filtering in radio engineering.

\section{Copyrights}

Copyright for this article is retained by the author(s), with first publication rights granted to the journal.

This is an open-access article distributed under the terms and conditions of the Creative Commons Attribution license (http://creativecommons.org/licenses/by/3.0/). 